\title{
Failure of Glidescope, rescue by macintosh-Old is undoubtedly Gold !
}

Editor - The past few years have witnessed an increasing use of videolaryngoscopy for the management of the patients with difficult intubation. Glidescope video laryngoscope has recently gained popularity for the management of difficult airway. ${ }^{1}$

We recently encountered a failed intubation using glidescope in a patient with rheumatoid arthritis. A 51 year old woman, height $155 \mathrm{~cm}$, weight $60 \mathrm{~kg}$ was posted for bilateral percutaneous nephrolithotripsy. She had a Mallampati score IV, mouth opening of 2 fingers, thyro mental distance of $4 \mathrm{~cm}$ and restricted neck movements. Intubation using Glidescope video laryngoscope was kept as Plan A for intubation. Anaesthesia was induced with standard general anaesthesia protocol and intubation was attempted using size 4 blade of Glidescope GVL. Only epiglottis was visible. After application of external laryngeal manipulation, the Cormack -Lehane grading improved to IIb. Endotracheal intubation using a $7 \mathrm{~mm}$ ID endotracheal tube, mounted on the angulated stylet was tried, but failed. Repeated attempts lead to trauma of the oropharyngeal cavity and the vision was obscured due to bleeding. After suctioning the oropharyngeal cavity, direct laryngoscopy using Macintosh laryngoscope was attempted. A $7 \mathrm{~mm}$ ID endotracheal tube was successfully passed over a gum elastic bougie. Between the intubation attempts, the patient was ventilated and arterial oxygen saturation was maintained above $90 \%$. The surgery was uneventful and the patient was extubated when fully awake.

Videolaryngoscopes undoubtedly improve the glottic visualization; however difficulty can be encountered during intubation of the trachea. Similar problem was faced in our patient in spite of a good laryngoscopic view.

Till the predictive criteria for difficult videolaryngoscopy are established unanticipated failure to intubate are bound to occur. The efficacy of the different video laryngoscopes in difficult airway situations need to be evaluated, before they gain a position in the difficult airway algorithm. ${ }^{2}$
D Jain, I Bala, K Sharma.

Department of Anaesthesia and Intensive Care

Postgraduate Institute of Medical Education and Research, Chandigarh, India.

\section{References}

1. Malik MA, Subramaniam R, Maharaj $\mathrm{CH}$, Harte BH, Laffey JG: Randomized controlled trial of the Pentax AWS ${ }^{\circledR}$, Glidescope ${ }^{\circledR}$, and Macintosh laryngoscopes in predicted difficult intubation.Br J Anaesth 2009; 103:761-8

http://dx.doi.org/10.1093/bja/aep266

PMid:19783539

2. Asai T. Videolaryngoscopes- Do They Truly Have Roles in Difficult Airways?

Anesthesiology 2012; 116:515-7. http://dx.doi.org/10.1097/ALN.0b013e318246e $\underline{866}$

PMid:22261794 